\title{
La incidencia del género en la prevención de riesgos laborales
}

The incidence of gender in the prevention of work risks

\author{
Dra. Ana Isabel PÉREZ CAMPOS \\ Universidad Rey Juan Carlos \\ Madrid
}

Resumen: Las distintas manifestaciones del género han tenido y mantienen un especial efecto en el ámbito de la prevención de riesgos laborales. La realidad actual muestra que teóricamente hombres y mujeres están sometidos al mismo riesgo laboral, pero en la práctica persisten las diferencias, porque hombres y mujeres se enferman o accidentan de forma diferente.

Partiendo de este diagnóstico, se pretenden valorar la influencia que la perspectiva de género tiene en la prevención de riesgos laborales, así como valorar el contenido, alcance y eficacia del nivel de protección y prevención, para, posteriormente, formular propuestas para un futuro desarrollo normativo y/o convencional.

Abstract: The different manifestations of the genre have had and continue to have a special impact in the field of occupational risk prevention. The current reality shows that theoretically men and women are subject to the same occupational risk, but in practice the differences persist, because men and women get sick or have accidents in different ways.

Based on this diagnosis, the aim is to assess the impact that the gender perspective has on the prevention of occupational hazards, as well as to assess the content, scope and effectiveness of the level of protection and prevention, to subsequently formulate proposals for future regulatory development and / or conventional.

Palabras clave: Género, seguridad y salud, protección y prevención

Keywords: Génder, safety and health, protection and prevention 
Sumario:

I. Introducción.

II. El género en el contexto de la seguridad y salud en el trabajo. Factores de riesgo laboral.

2.1. Envejecimiento.

2.2. Segregación horizontal y vertical.

2.3. Riesgos psicosociales y roles de género.

III. La protección de la prevención de riesgos laborales con perspectiva género.

3.1. Protección en el ámbito internacional y europeo.

3.2. Protección en España.

IV. Propuestas para un futuro desarrollo normativo y convencional.

4.1. La integración de la perspectiva de género en la prevención de riesgos laborales

4.2. La negociación colectiva como herramienta clave para su implantación.

V. Consideraciones finales.

VI. Bibliografía.

Recibido: noviembre de 2020.

Aceptado: enero de 2021. 


\section{INTRODUCCIÓN}

La variable género tiene una especial incidencia en el ámbito de la prevención de riesgos laborales en general y, en particular, frente a los riesgos profesionales, toda vez que los hombres y las mujeres se enferman o accidentan de diferente forma.

Si bien es cierto que las cuestiones de género no tienen un carácter innovador en el ámbito de la prevención de riesgos laborales, en la medida en que son múltiples los estudios que, desde el derecho y otras ciencias sociales, han tratado de resaltar las circunstancias específicas que afectan a las mujeres en su condición de trabajadoras ${ }^{1}$, la realidad actual muestra que aunque teóricamente hombres y mujeres están sometidos al mismo riesgo laboral, en la práctica hay diferencias importantes, es decir, existe cierta relación entre la feminización y los mencionados riesgos; distinción, además, que resulta ser mucho más acentuada en el ámbito de los riesgos profesionales.

\footnotetext{
${ }^{1}$ Entre otros, véanse: AA.VV., Informe sobre salud laboral desde la perspectiva de género, Dir. E. Blázquez Agudo, Instituto Estudios de Género. Universidad Carlos III de Madrid. Getafe 2017. GONZÁLEZ GÓMEZ, Ma F., "Salud laboral y género. Apuntes para la incorporación de la perspectiva de género en el ámbito de la prevención de riesgos laborales", en Revista medicina y seguridad en el trabajo, 57 (2011) 89-11. MARTÍNEZ YÁÑEZ, N.Mª., "Algunas consideraciones sobre igualdad por razón de género en el marco de la seguridad y salud en el trabajo", en Lan Harremanak, 25 (2012)155-189. FERNÁNDEZ DOCAMPO, B., "La salud laboral desde una perspectiva de género", en AA.VV., El principio de igualdad en la negociación colectiva", Dir. J.F. Lousada Arochena, 2008, p. 325. OLARTE ENCABO, S., "La discriminación en materia de prevención de riesgos laborales, en AA.VV., El principio de igualdad en la negociación colectiva, (Dir. C. Sánchez Trigueros), Ministerio de Empleo y Seguridad Social, Madrid 2016, pp. 375-390. RIVAS VALLEJO, P., "Salud y género: perspectiva de género en la salud laboral", en Revista del Ministerio de trabajo y asuntos sociales, 74 (2008). RIVAS VALLEJO, P., "La prevención de riesgos desde una perspectiva de género: posibilidades y realidades". Anuario de relaciones laborales en España: objetivo el trabajo, coord. por Antonio Ferrer Sais; Santos M. Ruesga Benito (ed. lit.), Carlos Resa Nestares (ed. lit.), 2 (2011), 253-255.

Documento de la Secretaría de Salud Laboral y Medio Ambiente de UGT-CEC titulado La perspectiva de género en la prevención de Riesgos Laborales depósito Legal: M-11282-2019.

http://www.observatorioriesgospsicosociales.com/sites/default/files/publicaciones/La\%20 Perspectiva\%20de\%20Genero.pdf.
} 
Así pues, a pesar de que las estadísticas nos revelan que el porcentaje de hombres que sufren accidentes laborales graves ha sido y es más elevado que el de las mujeres, éstas sufren un mayor número de enfermedades profesionales y también un elevado porcentaje -superior al del hombre- en accidentes in itinere. Las mujeres tienen más probabilidades de padecer problemas de salud relacionados con el trabajo que los hombres; sin embargo, los riesgos a los que se exponen las mujeres en el lugar de trabajo siguen siendo a menudo ignorados.

Teniendo en cuenta estas premisas, este estudio tiene como objetivo analizar la prevención de riesgos laborales desde una perspectiva de género. Se pretende reflexionar acerca del contenido, alcance y eficacia del nivel de protección y prevención y, posteriormente, formular propuestas para un futuro desarrollo normativo y/o convencional.

\section{EL GÉNERO EN EL CONTEXTO DE LA SEGURIDAD Y SALUD EN EL TRABAJO: FACTORES DE RIESGO LABORAL}

Desde la década de 1960, la esperanza de vida ha ido en aumento en el conjunto de Europa, al tiempo que se ha mantenido una baja tasa de natalidad. La población europea está envejeciendo y se espera que para 2040 los mayores de 65 años representen cerca del $27 \%$ de la población. Aunque la esperanza de vida también aumenta, en esos años de vida adicionales no necesariamente se goza de buena salud y la incidencia de enfermedades crónicas -cáncer, trastornos musculo-esqueléticos, enfermedades cardiovasculares, diabetes, depresión, etc.aumentan con la edad ${ }^{2}$.

El alargamiento de la esperanza de vida, el descenso de las tasas de natalidad, la incorporación tardía de la mujer al mercado de trabajo y el retraso en la edad de jubilación están produciendo una ampliación de la vida laboral que puede desarrollarse en algunos casos bajo circunstancias especialmente traumáticas para las mujeres trabajadores. Por ello, se hace necesario análisis sincrónico del género en el marco de la prevención de riesgos laborales, toda vez que la productividad y el rendimiento, deben ir en paralelo a la salud, la satisfacción y, en definitiva, una mejor calidad de vida.

Un trabajador puede verse expuesto a diferentes riesgos que, además, pueden verse agravados por factores tales como el género, el estado físico, o la edad. Adaptar el trabajo a las características individuales de cada trabajador es fundamental para favorecer un entorno laboral seguro y más saludable y,

\footnotetext{
${ }^{2}$ COPSEY. S., "Trabajo más seguro y saludable a cualquier edad", en AA.VV., El Envejecimiento de la Población Trabajadora Balance crítico de la situación y propuestas de mejora. Observatorio Vasco sobre Acoso y Discriminación, Bizkaia, 2018, pp. 325-335.
} 
como veremos, éste es uno de los retos preventivos que urge abordar para avanzar en la mejora de la prevención.

Al margen de las diferencias físicas y biológicas entre hombres y mujeres, existen otros factores que también separan a ambos colectivos y que, por su repercusión en el bienestar físico e incluso psicológico y su gran impacto en su salud, deberían ser tenidos en cuenta a la hora de regular y ordenar jurídicamente la prevención de riesgos laborales. En la mayoría de los supuestos, se trata de circunstancias de índole social y laboral, constitutivas de los llamados "riesgos psicosociales", de marcado carácter invisible al no ser tenidos en cuenta por la sociedad como un problema colectivo, sino más bien un problema individual perteneciente a la esfera privada de las personas.

La realidad muestra que, aunque teóricamente los hombres y las mujeres están sometidos al mismo riesgo laboral, en la práctica hay diferencias importantes ${ }^{3}$. Las mujeres tienen más probabilidades de padecer problemas de salud relacionados con el trabajo prolongado en el tiempo; por ejemplo, los trastornos musculoesqueléticos, en especial de las extremidades superiores, tienen una importante incidencia en el colectivo femenino, pues muchos de los trabajos realizados por mujeres requieren posturas forzadas, consisten en tareas monótonas y repetitivas, en manipulación de cargas o implican un gran esfuerzo ${ }^{4}$.

Por otra parte, sectores profesionales y condiciones de trabajo diferentes implican factores de riesgo también diferentes: distintas tareas, interacción persona/puesto, duración de la exposición al riesgo, etc., de forma que a pesar de que las condiciones de empleo sean aparentemente las mismas para hombres y mujeres, su repercusión sobre la salud de los trabajadores puede ser distinta en función del género.

Teniendo en cuenta que por factor de riesgo se puede entender el aspecto de la concepción, organización y gestión del trabajo, así como de su contexto social y ambiental que tiene la potencialidad de causar daños físicos, sociales o psicológicos en los trabajadores, a nuestros efectos, tendremos oportunidad de demostrar que determinados factores asociados al género -edad, segregación y roles- se constituyen en factores de riesgo laboral.

${ }^{3}$ GIL PÉREZ, M. E., "Mujer y salud laboral”, en AA.VV., Mujer y trabajo, Bomarzo, Albacete 2003, pp. 129-156. PÉREZ DEL RÍO, T., y BALLESTER PASTOR, M. A., Mujer y salud laboral, La Ley, Las Rozas, Madrid 1999, p.1.

${ }^{4}$ MARTÍNEZ YÁÑEZ, N., "Algunas consideraciones sobre igualdad por razón de género en el marco de la seguridad y salud en el trabajo", en Lan Harremanak. Revista de Relaciones Laborales, 25 (2011)161. 


\subsection{Envejecimiento}

El envejecimiento de la población es una realidad demográfica que afecta a la sociedad actual, constituyendo uno de los mayores cambios y desafíos de nuestro tiempo. En este factor demográfico coinciden dos fenómenos: por una parte, una mayor esperanza de vida, aún desconocidos los límites de ese aumento; y, por otra, el bajo índice de natalidad, en general, en toda Europa. Casi con toda probabilidad, el incremento del índice de natalidad no será suficiente para frenar la aceleración del envejecimiento; sin embargo, los costes que generará dicho envejecimiento no corresponden solamente a un mayor gasto en pensiones, sino también en lo que respecta a los sistemas de protección social, de seguridad y salud de los trabajadores.

Como se ha señalado anteriormente, los accidentes en jornada de los hombres, se sitúan en cifras superiores a la de las mujeres, para todos los grupos de edad ${ }^{5}$. Por tanto, el paso de los años, que implica madurez y veteranía, tiene un efecto beneficioso al reducir la accidentalidad de los trabajadores. Por el contario, la edad "per se" no es un factor relevante en la accidentalidad en jornada de las trabajadoras. Ahora bien, la población laboral de mayor edad, a pesar de la experiencia acumulada, puede ser más vulnerable a determinados riesgos, en gran parte influida por los cambios fisiológicos derivados del envejecimiento y del efecto de las enfermedades crónicas.

Los trabajadores de edad avanzada son los que sufren accidentes más graves, con una tasa de mortalidad superior a la media ${ }^{6}$. El promedio de edad de accidentarse o enfermarse en hombres es de 37 años, mientras que en las mujeres asciende hasta los 40 años. Asimismo, el $80 \%$ de los casos notificados de mujeres corresponde a edades entre 25 y 54 años, mientras que en el $75 \%$ de los casos en los que el accidentado fue un hombre, las edades oscilan entre los 20 y 44 años ${ }^{7}$.

\footnotetext{
${ }^{5}$ La siniestralidad con accidente mortal es más frecuente en los varones a partir de los 45 años, mientras que las mujeres tienen una baja siniestralidad laboral con resultado de muerte, con independencia de la edad. En los grupos de menor edad (de 16 a 29 años) la diferencia de índices entre hombres y mujeres es elevada, siendo la siniestralidad de los varones más del doble que la de las mujeres. Véase, Informe anual de accidentes de trabajo en España elaborado por el INSST elaborador a partir del anuario de Estadísticas del Ministerio de Trabajo, Migraciones y Seguridad Social 2019.

http://www.mites.gob.es/ficheros/ministerio/estadisticas/anuarios/2019/EPR/EPR.pdf.

${ }^{6}$ Instituto Nacional de Seguridad e Higiene en el Trabajo (INSHT). Estrategia española de seguridad y salud en el trabajo. 2015-2020. Madrid, abril 2015.

${ }^{7}$ Estadística de accidentes de trabajo del Ministerio de Trabajo y economía social.

http://www.mites.gob.es/estadisticas/eat/eat20_06/ATR_06_2020_Resumen.pdf.
} 
Según las cifras facilitadas por el Ministerio de Trabajo y Economía Social durante 2019 y centrando el análisis en los accidentes con baja ocurridos durante la jornada laboral, el $74 \%$ de los accidentes de trabajo con baja se registraron entre varones frente al $26 \%$ que corresponde a mujeres ${ }^{8}$.

En las pensiones derivadas de enfermedad profesional, existe una mayor dispersión en las franjas de edad, así como un menor número de prestaciones reconocidas. Por ejemplo, la franja de edad donde se reconoció el mayor número de pensiones por esta causa, se sitúa entre los 45 y los 49 años y en un porcentaje mayor para los trabajadores masculinos. En cuanto a las edades medias, la de las mujeres pensionistas es ligeramente superior a la de los hombres, al situarse en 57 años, frente a los 53 del segundo caso. Si bien en las edades superiores a los 65 años, se invierten las cifras mucho más elevadas en número de pensionistas por sexos, que pasan de ser prioritariamente masculinas a femeninas, dada la mayor esperanza de vida de la mujer?.

En consecuencia, desde una perspectiva de género, la evolución de la siniestralidad nos indica que el perfil del accidente en jornada de trabajo es marcadamente masculino y se manifiesta al alza.

Por otra parte, resulta muy interesante el dato de accidentes de trabajo in itinere, donde los accidentes del colectivo femenino alcanzan el $54 \%$, por tanto, superior al de los varones. Se trata de una tendencia, que no ha sufrido una evolución ascendente -al mantenerse en porcentajes anuales similares desde 2012-. Ahora bien, es necesario precisar a este respecto que la siniestralidad mortal in itinere tiene un perfil masculino; probablemente asociado al hecho del mayor uso por parte de los varones del automóvil particular, como modo de desplazamiento al trabajo ${ }^{10}$.

En definitiva, los accidentes de trabajo in itinere, son en número "femeninos" y en gravedad "masculinos. La siniestralidad con accidente mortal es más frecuente en los varones a partir de los 45 años, mientras que las mujeres tienen una baja siniestralidad laboral con resultado de muerte, con independencia de la edad.

${ }^{8}$ Ibidem.

${ }^{9}$ Ibidem. Esta distinción no es nueva, se ha venido manifestando desde hace tiempo. Al respecto, véase el estudio realizado por RIVAS VALLEJO, P., "Salud y género: perspectiva de género en la salud laboral"..., o.c., p. 268.

${ }^{10}$ Informe anual de accidentes de trabajo en España elaborado por el INSST, elaborador a partir del Anuario de Estadísticas del Ministerio de Trabajo, Migraciones y Seguridad Social 2019. https://www.insst.es/documentacion/catalogo-de-publicaciones/informe-anual-de-accidentesde-trabajo-en-espana. 
En el caso de las enfermedades profesionales los datos reflejan otra realidad, en la medida en que la evolución no muestra un patrón claro de crecimiento diferenciado de las enfermedades profesionales de las mujeres. Los últimos datos obtenidos sobre enfermedades profesionales según sexo, sector y actividad son de 2019 y reflejan que se produjeron un total de 27.292, de las cuales 13.257 son de varones frente a 14.035 de mujeres ${ }^{11}$. Las enfermedades profesionales son más frecuentes en las mujeres en número, pero no en gravedad. La siniestralidad en el trabajo de las mujeres representa el $32,34 \%$ del total de los procesos vinculados con contingencias profesionales. Con relación al sexo, el número de enfermedades notificadas ha sido mayor en hombres que en mujeres, en una proporción de 2 a 1 . A nivel de gravedad de los accidentes de trabajo y las enfermedades profesionales, la gran mayoría, han sido catalogados como leves, unos pocos han sido valorados como graves y la cifra más baja ha sido para los accidentes mortales ${ }^{12}$.

En consecuencia, los hombres tienen más probabilidades de sufrir accidentes laborales que las mujeres. Las mujeres tienen más probabilidades de padecer problemas de salud relacionados con el trabajo que los hombres. Sin embargo, los riesgos a los que se exponen las mujeres en el lugar de trabajo siguen siendo a menudo ignorados.

\subsection{Segregación horizontal y vertical}

Trabajar en distintos sectores y ocupaciones -segregación horizontal- o, en el mismo sector, pero realizando diferentes tareas o tareas inferiores -segregación vertical-, producen desigualdades que tienen un reflejo exponencial en el ámbito de la seguridad y la salud. Los trabajos de las mujeres presentan unas características específicas: repetición, monotonía, esfuerzo estático, múltiples responsabilidades simultáneas que, con el tiempo, pueden afectar a la salud física y mental de la trabajadora.

11 En los últimos años, el número total de partes comunicados es superior en mujeres que en hombres (14.146 por 13.197), aunque este efecto ya se venía apreciando desde 2013 en el caso de los partes de enfermedad profesional con baja.

http://www.mites.gob.es/es/estadisticas/condiciones_trabajo_relac_laborales/EPR/welcome.htm.

Véase también, GARCÍA GÓMEZ, M.; CASTAÑ̃EDA LÓPEZ, R.; HERRADOR ORTIZ, Z.; LÓPEZ MENDUIÑA, P.; MONTOYA MARTÍNEZ, L.M.; ÁLVAREZ MAEZTU, E., et Al., Estudio epidemiológico de las enfermedades profesionales en España (1990 - 2014). Madrid, Ministerio de Sanidad, Servicios Sociales e Igualdad, 2017.

$12 \mathrm{http}: / / \mathrm{www} . \mathrm{mites}$.gob.es/es/estadisticas/condiciones trabajo relac laborales/EPR/welcome.htm.

Véase también GARCÍA GÓMEZ, M., y CASTAÑEDA LŌPEZ, R., "Enfermedades profesionales declaradas en hombres y mujeres en España en 2004”, en Revista Española de Salud Pública, 4 (2006). 
El reparto de trabajos entre hombres y mujeres por sectores de actividad explica una parte de las diferencias en la siniestralidad durante la jornada laboral, condicionando el tipo de accidente que se sufre en jornada, aunque también influye su rol social que provoca la necesidad de trabajar rápido o atender varias tareas, aumentando esta circunstancia de manera exponencial el riesgo laboral. Más allá de las desigualdades que todavía existen, la distribución y la concentración en diferentes actividades está estructuralmente determinada por el sexo.

Los hombres se concentran, principalmente, en sectores como la construcción, minería, manufacturas, transporte y agricultura, mientras que las mujeres tienden a agruparse en el sector de servicios, con el foco ubicado en sanidad y educación, de forma que esta distinción de tareas va a provocar una mayor siniestralidad para los hombres ya que están expuestos a muchos más riesgos, ambientes insalubres y material tóxico. La inserción de la mujer trabajadora en los sectores masculinizados es poco significativa ${ }^{13}$.

${ }^{13}$ El Informe del Observatorio de las ocupaciones, Informe del mercado de trabajo de las mujeres de 2018, servicio público de empleo estatal 2019 muestra una evidente segmentación por sexos en el mercado laboral muestra una evidente segmentación por sexos en el mercado laboral. No obstante, se observa un aumento de la contratación femenina en todos los sectores económicos. El mayor incremento interanual se produjo en la industria, donde la representación de la contratación a mujeres experimentó un ligero avance. Las actividades con mayor aceleración con respecto al año anterior fueron Actividades de servicios sociales sin alojamiento; Actividades sanitarias; Servicios a edificios y actividades de jardinería; Asistencia en establecimientos residenciales; y Educación.

Por otra parte, la publicación por parte del INE de fichas asociadas a indicadores del INE, en concreto la realizada a empleo de hombres y mujeres de 2020 , dentro de los datos sobre ocupación por rama de actividad destaca que en el año 2019 del total de mujeres ocupadas, el porcentaje más alto de participación $(16,7 \%)$ por rama de actividad económica corresponde a la actividad G. Comercio al por mayor y al por menor, reparación de vehículos de motor y motocicletas. El segundo lugar (14,2\%) corresponde a la actividad Q. Actividades sanitarias y de servicios sociales, y el tercer lugar a la actividad I. Hostelería y a la actividad P. Educación, ambas con $(10,2 \%)$. Los porcentajes más bajos de actividad femenina corresponden a las ramas: D. Suministro de energía eléctrica, gas, vapor y aire acondicionado, con un $0,3 \%$ de mujeres empleadas y E. Suministro de agua, actividades de saneamiento, gestión de residuos y descontaminación $(0,3 \%$ de mujeres empleadas). En las ramas de actividad B. Industrias extractivas y U. Actividades de organizaciones y organismos extraterritoriales, no hay participación femenina. En el año 2019, en el total de mujeres ocupadas el porcentaje más alto $(29,0 \%)$ correspondía a la ocupación 5 . Trabajadores de los servicios de restauración, personales, protección y vendedores. El segundo lugar $(22,8 \%)$ a la ocupación 2 . Técnicos y profesionales científicos e intelectuales y el tercer lugar $(16,1 \%)$ a la ocupación 9 . Ocupaciones elementales. Los porcentajes más bajos correspondían a la ocupación 0 . Ocupaciones militares $(0,1 \%)$, y a la ocupación 6 . Trabajadores cualificados en el sector agrícola, ganadero, forestal y pesquero.

https://www.ine.es/ss/Satellite?L=es_ES\&c=INESeccion_C\&cid=1259931459725\&p=12 54735110672 \&pagename=ProductosYServicios\%2FPYSLayout\&param1=PYSDetalle\&para $\mathrm{m} 3=1259924822888$. 
Por otra parte, las mujeres se concentran en torno a unas actividades, dentro de las cuales se produce el mayor número de enfermedades profesionales. Así, superan a los hombres en los sectores de hostelería, actividades inmobiliarias y de alquiler, servicios empresariales, educación, actividades sanitarias y veterinarias, servicios sociales, y en el servicio doméstico. Dentro de estas actividades resulta incluso lógico que el número de mujeres siniestradas sea superior al de hombres ${ }^{14}$.

En los trabajos ocupados mayoritariamente por mujeres predominan los riesgos relacionados con la organización del trabajo -las posturas de trabajo inadecuadas, largas jornadas de pie, trabajos repetitivos, manipulación de numerosos objetos de poco peso y riesgos, entre otros- y producen daños que aparecen de manera lenta y progresiva, como son las lesiones en el cuello, en los brazos y alteraciones de la salud psíquica, no reconocidas legalmente como enfermedades profesionales y, por tanto, tampoco computan para las cifras globales de siniestralidad, ni tienen cobertura de protección social ${ }^{15}$.

La menor visibilidad de la siniestralidad de las mujeres es provocada, entre otros factores, por el hecho de que casi nunca es mortal, aspecto éste que puede haber contribuido a su subestimación e, incluso invisibilidad. La prevención de riesgos laborales tradicionalmente ha girado en torno a la seguridad industrial, la higiene en el trabajo y la medicina del trabajo, orientadas todas ellas hacia la prevención de los accidentes de trabajo y las enfermedades profesionales de signo masculino ${ }^{16}$.

En consecuencia, las diferencias en los perfiles de riesgo laboral no derivan del sexo, sino de la segregación laboral que condiciona las cifras sobre siniestralidad laboral y que se traduce en cifras que lejos de ir reduciéndose parecen afianzarse.

\subsection{Riesgos psicosociales y roles de género}

Dentro de lo que la doctrina científica ha calificado como "nuevas enfermedades profesionales"17, se sitúan los riesgos psicosociales, emergentes en los últimos tiempos y objeto de una atención cada vez más acusada. Las mujeres son

14 GONZÁLEZ SEGARRA, F.J., Riesgos ergonómicos y psicosociales en los sectores feminizados de Andalucía. Ed. UGT-Andalucía, Sevilla 2006. ACALE SÁNCHEZ, M., "Género y siniestralidad laboral: causa y efecto", en Gestión Práctica de Riesgos Laborales, 69 (2010) 27.

${ }^{15}$ GONZÁLEZ SEGARRA, F.J., "Riesgos ergonómicos y psicosociales”..., o.c., p. 39.

${ }^{16}$ MARTÍNEZ YÁÑEZ, N., "Algunas consideraciones sobre igualdad por razón de género en el marco de la seguridad y salud en el trabajo" ..., o.c., p. 162.

${ }^{17}$ RIVAS VALLEJO, P., "Salud y género: perspectiva de género en la salud laboral" ..., o.c., p. 246. 
más vulnerables que los hombres a los riesgos de tipo psicológico, especialmente al estrés y la depresión y, en menor, medida al burnout, aunque son muy difíciles de valorar en las evaluaciones de riesgos ${ }^{18}$. Las consecuencias para la salud son la aparición de enfermedades coronarias, hipertensión, alteraciones del sueño, debilitamiento del sistema inmunológico, dependencia de drogas o sustancias farmacológicas, entre otros ${ }^{19}$. El empeoramiento de las condiciones de trabajo genera sobrecarga, estrés, presiones de tiempo, dificulta la conciliación de la vida laboral y familiar y todo esto repercute negativamente en la salud de las trabajadoras, de ahí que se continúe reivindicando la necesidad de inclusión de los riesgos psicosociales como una enfermedad profesional ${ }^{20}$.

Por otra parte, los roles de género, esto es, la asunción por parte de las mujeres de mayores responsabilidades domésticas y familiares puede también tener una incidencia negativa en el ámbito de la salud y seguridad. Las mujeres siguen efectuando la mayor parte del trabajo no remunerado en el hogar, tareas domésticas, y de cuidado de los hijos y familiares-, incluso cuando trabajan a tiempo completo, lo que provoca un incremento en la carga global de trabajo y, por tanto, de la siniestralidad por riesgo físico y psicosocial, junto a otros factores como acoso, y discriminación que refuerzan la necesidad de valorar el riesgo psicosocial. A mayor abundamiento, son empleos que, en muchos casos, constituyen una prolongación del trabajo reproductivo (cuidado de personas, educación, vestido y alimentación, etc.) lo que supone que sus efectos en la salud pueden ser más perniciosos en mujeres que en hombres, en parte por la suma de tiempos de trabajo -remunerado y reproductivo- en el mismo tipo de actividad.

Las denominadas tradicionalmente como ocupaciones feminizadas se caracterizan justamente por la presencia de este tipo de riesgos, casi siempre vinculados a la organización del trabajo y a la ergonomía, y con una escasa incidencia en la salud de las afectadas, en muchas ocasiones porque su efecto es lento y retardado.

Partiendo de este diagnóstico, habría que valorar los posibles peligros que para el trabajo genera la condición femenina para la seguridad y la salud, en

${ }^{18}$ BLÁZQUEZ AGUDO, E., "La necesaria asunción de la prevención de riesgos laborales desde una perspectiva de género", en Femeris, 5 (2020) 5.

19 Véase, al respecto, estudio de MARTÍNEZ YÁÑEZ, N., "Algunas consideraciones sobre igualdad por razón de género en el marco de la seguridad y salud en el trabajo"..., o.c. pp. 159-164.

${ }^{20}$ UGT. Guía de Calificación jurídica de las patologías causadas por Riesgos Psicosociales e. el trabajo. Propuesta de mejora. 2018.

https://www.ugt.es/sites/default/files/guiapatologiasweb.pdf 
la medida en que sigue siendo una cuestión esencialmente vinculada al género $^{21}$.

\section{LA PROTECCIÓN DE LA PREVENCIÓN DE RIESGOS LABO- RALES CON PERSPECTIVA DE GÉNERO}

La prevención de riesgos laborales con perspectiva de género es un fenómeno que ha adquirido gran relevancia tanto en el ámbito comunitario como internacional y que se ha visto fortalecido, durante los últimos años, donde la incidencia de los riesgos laborales y el género está siendo especialmente relevante.

\subsection{Protección en el ámbito internacional y europeo}

Las normas internacionales han sido pioneras en tratar la seguridad y salud laboral de las mujeres, aunque lo hacían pensando en políticas de prevención estrictamente biológicas y, por tanto, centradas en el embarazo y la maternidad, sin prestar la atención necesaria a otros aspectos de las condiciones laborales que también pueden tener un importante impacto en el ámbito de la seguridad y salud.

La incorporación de la mujer al mercado de trabajo provocó que se tomase conciencia de la relevancia que, a efectos de seguridad y salud, ostenta la perspectiva de género en su vertiente de protección frente a la maternidad, sin embargo, la vinculación directa del género y la prevención de riesgos laborales no es objeto de protección jurídica específica.

El concepto de perspectiva de género fue introducido oficialmente en la Conferencia Mundial de las Mujeres de Naciones Unidas en Beijing en 1995, donde se planteaba la necesidad de utilizar unas "gafas" especiales para valorar las diferentes situaciones, condiciones y posiciones de mujeres y hombres en la sociedad, en el mercado laboral, en la política, etc. y se definía como el proceso mediante el cual se valoran las implicaciones para las mujeres y los hombres de cualquier acción planificada, incluyendo legislaciones, políticas o programas en todas las áreas y todos los niveles ${ }^{22}$.

${ }^{21}$ FERNÁNDEZ DOCAMPO, B., "La salud laboral desde una perspectiva de género", en AA.VV., El principio de igualdad en la negociación colectiva" (Dir J.F. Lousada Arochena), 2008, p. 325. NIETO ROJAS, P., "Género, Prevención de Riesgos y Negociación colectiva", en Revista de información laboral, 9 (2017). (BIB 2017\13141).

${ }^{22} \mathrm{https}$ //www.un.org/womenwatch/daw/beijing/pdf/Beijing\%20full\%20report\%20S.pdf. 
En un sucinto repaso a la protección jurídica de los riesgos laborales y el género cabe destacar en el ámbito internacional, los convenios de la OIT que han sido la fuente inspiradora de las estructuras y de los contenidos de los sistemas nacionales de protección social, tanto de los cambios introducidos por los países con una Seguridad Social evolucionada como para los que han comenzado a organizarla o están empeñados en el esfuerzo de ampliarla y desarrollarla. Como se ha señalado, por este motivo los Convenios de la OIT siguen siendo valiosos y útiles ${ }^{23}$.

Como ejemplo de lo señalado, destaca el Convenio núm. 155 de la OIT sobre salud y seguridad en el trabajo de 1981, que además de relacionar los factores de riesgo, situaciones y condiciones que deben tenerse en cuenta para prevenir daños a la salud, como grandes esferas de acción de la política en materia de seguridad y salud laboral destaca la protección de los trabajadores y las trabajadoras y de quienes les representan contra toda medida disciplinaria que resulte de acciones emprendidas con motivo de protegerse frente a accidentes o daños a la salud ${ }^{24}$.

Desde una perspectiva europea, la prevención de riesgos laborales y el binomio igualdad y salud laboral presenta especialidades debidas tanto a los diferentes rasgos biológicos de los trabajadores masculinos y femeninos, como a otros aspectos sociales relacionados con el mercado de trabajo. Esta circunstancia provocó que en la Unión Europea se estableciesen planes y estrategias de igualdad y de prevención de riesgos donde se potenciaba el tener en cuenta las diferencias derivadas, directa o indirectamente, del sexo de las personas; aunque conviene precisar que estos planes o estrategias no convergieron en una única propuesta, sino que se diseñaron estrategias de igualdad y de salud y seguridad en el trabajo, separadas ${ }^{25}$.

La necesidad de contemplar las especificidades del género en materia de prevención de riesgos, en garantía y eficacia de igualdad, se ha puesto de manifiesto en determinados planes o estrategias de igualdad. Así, la Estrategia comunitaria para la igualdad entre mujeres y hombres (2010-2015) subrayó que las mujeres y los hombres se enfrentan a riesgos sanitarios, enfermedades, problemas y prácticas específicas que repercuten de forma diferente sobre su

${ }^{23}$ GONZALEZ ORTEGA, S., y BARCELÓN COBEDO, S., "Cien años de promoción de la Seguridad Social. El papel de la OIT en la creación, expansión, perfeccionamiento y adaptación de los sistemas de protección social", en Revista Trabajo y Derecho, 9 (2019).

${ }^{24} \mathrm{https} / / / w w w . i l o . o r g / d y n /$ normlex/es/f?p=NORMLEXPUB:12100:0::NO::P12100_ILO_ CODE:C155.

${ }^{25}$ MARTÍNEZ YÁÑEZ, N., "Algunas consideraciones sobre igualdad por razón de género en el marco de la seguridad y salud en el trabajo"..., o.c., p. 158. 
salud, y sin embargo, la investigación médica y muchas normas de seguridad y sanidad se centran más en los hombres y en los sectores predominantemente $\operatorname{masculinos}^{26}$; lo que propició la conveniencia de desarrollar un enfoque de la prevención de riesgos laborales diferenciado en función del sexo.

La Estrategia comunitaria de salud y seguridad en el Trabajo (2007-2012) partió de la idea de que las desigualdades pueden afectar a la salud y la seguridad de las mujeres en el trabajo e influir en su productividad. Además, añade que las diferencias por razón de género que inciden en los riesgos profesionales no sólo tienen su razón de ser en aspectos biológicos, sino que la situación del empleo y la mujer constituye también una importante traba en la consecución de la igualdad ${ }^{27}$.

Es imprescindible mencionar también que la Estrategia europea de seguridad y salud en el trabajo (2014-2020) alude a varios retos, tales como la mejora de la prevención de las enfermedades relacionadas con el trabajo haciendo frente a los riesgos nuevos y emergentes, sin descuidar los que ya existen y tener en cuenta el envejecimiento de la mano de obra de la UE.

Por otra parte, teniendo en cuenta el envejecimiento constante y progresivo de los trabajadores de la UE, la Agencia Europea para la Seguridad y la Salud, en el Trabajo en el periodo 2016-2017, para dar respuesta a estos crecientes desafíos de salud y seguridad laboral, señala que es preciso proceder a una evaluación del riesgo teniendo en cuenta la diversidad y prestar atención a las especificidades de sexo, edad y demás características demográficas, si bien no deben formularse hipótesis únicamente sobre tal base ${ }^{28}$. La evaluación del riesgo debe tener en cuenta los imperativos laborales en relación con las capacidades del individuo y con su salud.

Especial mención merece dentro de las directrices señaladas por el Consejo de ministros de la Unión Europea para la Estrategia de salud y seguridad en

${ }^{26}$ Comunicación al Parlamento Europeo, al Consejo, al Comité Económico y Social Europeo y al Comité de las Regiones, de 21 de septiembre de 2010, COM (2010) 491 final.

${ }^{27}$ Comunicación de la Comisión al Parlamento europeo, al Consejo, al Comité económico y social y al Comité de las regiones. Mejorar la calidad y la productividad en el trabajo: estrategia comunitaria de salud y seguridad en el trabajo (2007-2012), de 21 de febrero de 2007. $\operatorname{COM}(2007) 62$ final.

https://eur-lex.europa.eu/LexUriServ/LexUriServ.do?uri=COM:2007:0062:FIN:ES:PDF.

${ }^{28}$ Comunicación de la Comisión al Parlamento Europeo, al Consejo, al Comité Económico y Social Europeo y al Comité de las Regiones sobre "Trabajo más seguro y saludable para todos - Modernización de la legislación y las políticas de la UE de salud y seguridad en el trabajo" de 10 de enero de 2017, $\operatorname{COM}(2017) 12$ final.

https://eur-lex.europa.eu/legal-content/ES/TXT/HTML/?uri=CELEX:52010DC0491\&from=ES. 
el trabajo para el período 2021-2027 medidas específicas de prevención de riesgos con perspectiva de género, al indicar que "Las mujeres trabajan a menudo en diferentes sectores en los que las condiciones de trabajo y los riesgos para la salud y la seguridad en el trabajo son diferentes de los de los hombres"29. A su vez, la Estrategia comunitaria para la igualdad de género (2020-2025), en la línea marcada por su precedente, solo alude a la prevención de riesgos y el género en relación con la maternidad, no obstante, sí que contempla una futura integración de la perspectiva de género en todos los ámbitos políticos, a todos los niveles y en todas las políticas de la Unión ${ }^{30}$.

Por otra parte, el principio de igualdad formal ha presidido de forma invariable la elaboración de las numerosas Directivas sobre seguridad y salud en el trabajo, que contienen disposiciones comunes para todos los trabajadores, ajenas a las diferencias materiales que puedan existir en la práctica.

Las directivas europeas sobre prevención de riesgos laborales suelen adoptar un enfoque neutro con respecto al género ${ }^{31}$. El único cambio significativo se refleja en el artículo 1 de la Directiva 2007/30/CE, del Parlamento Europeo y del Consejo, de 20 de junio, por la que se modifica la Directiva 89/391/CEE del Consejo, sus directivas específicas y las Directivas 83/477/CEE, 91/383/CEE, 92/29/CEE y 94/33/CE del Consejo, a fin de simplificar y racionalizar los informes sobre su aplicación práctica. Dicho precepto inserta en la Directiva 89/391/CEE un nuevo artículo 17 bis, en el que se establece la obligación de los Estados miembros de presentar cada cinco años a la Comisión un informe sobre la aplicación de varias Directivas de seguridad y salud en el trabajo en el que han de aparecer los datos desglosados por género, aunque matiza que sólo "cuando se disponga de los mismos y resulte procedente". La Directiva 2006/54/CE, relativa a la aplicación del principio de igualdad de oportunidades e igualdad de trato entre hombres y mujeres en asuntos de empleo y ocupación (refundición), refiere -en su art. 26- al establecimiento de una obligación preventiva relacionada con el sexo y el género, pero definitivamente ajena a la protección del proceso reproductivo: la "prevención" de la discriminación por razón de sexo y del acoso sexual y por razón de sexo en el trabajo en

${ }^{29}$ Véase, proyecto de conclusiones del Consejo de 5 de diciembre de 2019, sobre un nuevo marco estratégico de la UE en materia de salud y seguridad en el trabajo 2014-2020: mejorar la aplicación de la salud y la seguridad en el trabajo en la EU. ST 14256/19.

${ }^{30}$ Comunicación de la Comisión al Parlamento europeo, al Consejo, al Comité económico y social europeo y al Comité de las regiones. Una Unión de la igualdad: Estrategia para la Igualdad de Género 2020-2025, de 5 de marzo de 2020. COM(2020) 152 final. https://eur-lex.europa.eu/legal-content/ES/TXT/HTML/?uri=CELEX:52020DC0152\&from=ES

${ }^{31}$ Agencia Europea para la Seguridad y Salud en el trabajo. Las cuestiones de género en relación con la seguridad y la salud en el trabajo, Luxemburgo 2006, p. 142. 
tanto son riesgos laborales de naturaleza psicosocial, especialmente prevalentes entre las mujeres.

Una última referencia dentro del marco normativo de protección es el proyecto experimental "Safer and healthier work at any age" (Trabajo más seguro y saludable a cualquier edad), de la Agencia Europea para la Seguridad y la Salud en el Trabajo (EU-OSHA) que se ha centrado a través en las dificultades que plantea el envejecimiento de la población activa europea para la seguridad y la salud en el trabajo (SST) ${ }^{32}$.El citado informe menciona que resulta necesario prestar apoyo a las evaluaciones de riesgo que incorporen cuestiones relativas a la edad y el género; el desempeño de empleos de bajo nivel, sin promoción de la carrera profesional durante largos períodos, puede conducir a una exposición prolongada a peligros para la salud; la igualdad debe abordarse en el acceso a la rehabilitación y en la formación profesional. Así mismo, señala la necesidad de implementar estrategias de promoción de la salud en el lugar de trabajo, se exigen distintos enfoques en función de que los destinatarios sean hombres o mujeres. Las trabajadoras de más edad deben considerarse un activo valioso, y la doble discriminación a la que estas trabajadoras pueden enfrentarse debe ser abordada por medio del incremento de la sensibilización en este aspecto.

\subsection{Protección en España}

En el ordenamiento español destaca la Ley $31 / 2015$ de 8 de noviembre de prevención de riesgos labores ${ }^{33}$ que tiene por objeto promover la seguridad y la salud de los trabajadores mediante la aplicación de medidas y el desarrollo de las actividades necesarias para la prevención de riesgos derivados del trabajo. Más específicamente, se configura, como una referencia legal que establece un marco legal mínimo a partir del cual las normas reglamentarias irán fijando y concretando los aspectos más técnicos de las medidas preventivas; y segundo, como soporte básico a partir de la cual la negociación colectiva podrá desarrollar su función específica.

Por otra parte, el artículo 4, apartado quinto de la Ley de Prevención de Riesgos Laborales, alude a la obligación de las Administraciones Públicas de

32 EU-OSHA (2016), Women and the ageing workforce: implications for occupational safety and health - A research review (Revisión de la investigación sobre las mujeres y las consecuencias del envejecimiento de la población activa para la seguridad y la salud en el trabajo),

https://osha.europa.eu/es/tools-and-publications/publications/safer-and-healthier-work-any-agewomen-and-ageingworkforce/view.

${ }^{33}$ BOE 10 de noviembre de 2015. 
promover la efectividad del principio de igualdad entre mujeres y hombres, considerando las variables relacionadas con el sexo tanto en los sistemas de recogida y tratamiento de datos como en el estudio e investigaciones generales en materia de prevención de riesgos laborales.

La Estrategia Española de Seguridad y Salud en el Trabajo 2015-2020, enumera las prioridades para la mejora de las condiciones de seguridad y salud de los sectores con mayores riesgos, la especial atención a los trastornos musculo-esqueléticos -sin alusión a género-, la definición de trabajadores vulnerables y colectivos específicos entre los que se considera urgente una especial atención -jóvenes, trabajadores temporales, trabajadores mayores, de empresas de trabajo temporal y trabajadores autónomos-. No obstante, contempla referencias al género en sus objetivos donde destaca la necesidad de impulsar la integración de aspectos de género en la actividad preventiva, especialmente en sectores con mayor presencia de la mujer. Al margen de estas precisiones, las referencias a la problemática de la prevención de riesgos y el género son prácticamente inexistentes.

En definitiva, no existe una política preventiva específica de riesgos laborales desagregada por sexos desde el punto de vista del marco legal vigente, ni en las políticas internas de las empresas españolas, ni tampoco a nivel convencional. Y es que la política de protección y prevención está formulada en términos neutros sin tener en cuenta las especificidades del trabajo femenino, salvo para los aspectos especialmente relacionados con la maternidad.

\section{PROPUESTAS PARA UN FUTURO DESARROLLO NORMATIVO Y CONVENCIONAL}

El análisis de la incidencia de los riesgos laborales y el género obliga a reflexionar sobre el futuro de la protección social y de la seguridad y la salud en el trabajo y sobre las reformas a implementar, en aras, a la reducción o eliminación de las importantes brechas de género que aún tienen un lugar destacado. La combinación de características estructurales sin superar del pasado y la aparición de nuevos desafíos se convierten en complejos retos de protección en el ámbito de la seguridad y la salud. A partir de este diagnóstico, algunos autores aluden incluso a la discriminación en materia de prevención de riesgos laborales ${ }^{34}$.

\footnotetext{
34 "La discriminación por razón de género en la prevención de riesgos laborales adopta formas sutiles que constituyen, en su caso, formas de discriminación indirecta....que consistiría, no en la exclusión de las mujeres de las medidas preventivas, sino en la no consideración de lo diferencial de la mujer frente al hombre a la hora de adoptar medidas de prevención de riesgos
} 
La erradicación de los riesgos laborales que afectan en mayor medida a las mujeres no puede llevarse a cabo sólo desde una perspectiva estrictamente preventiva, sino que debe ser objeto de un abordaje global por parte del ordenamiento jurídico. Las medidas para atajar la discriminación salarial, para reforzar las oportunidades de empleo de las mujeres, para fomentar la corresponsabilidad entre los sexos, la efectiva conciliación de la vida laboral y familiar, así como una especial protección frente a los riesgos psicosociales, entre otras, son claves para garantizar la seguridad y salud de las trabajadoras.

\subsection{La integración de la perspectiva de género en la prevención de riesgos laborales}

La integración del género en las cuestiones de protección y prevención de la seguridad y salud en el trabajo constituye un elemento importante de mejora en los riesgos laborales, pero también una condición necesaria para el desarrollo económico y social ${ }^{35}$. A pesar de los esfuerzos del legislador hacia la efectividad de la igualdad en el empleo y la ocupación, estos esfuerzos no han dado los resultados esperados.

En lo que refiere a la dualidad género-riesgo laboral, si bien es cierto que la insuficiente protección jurídica pueda ser una de las causas de que no se haya podido garantizar la igualdad de trato, no debe olvidarse que la atención de las normas sobre prevención de riesgos laborales, por lo que se refiere a la seguridad y salud de las mujeres en el trabajo, se ha circunscrito tradicionalmente al abordaje de los riesgos laborales relacionados con la maternidad.

En consecuencia, resulta necesario dar un nuevo enfoque a la protección de riesgos laborales, en particular a los riesgos profesionales, a través de la inclusión de normas con dimensión de género. Como se viene reivindicando a nivel internacional, es preciso tener en cuenta los factores específicos de género en el contexto de las normas en materia de seguridad y salud en el trabajo ${ }^{36}$.

\footnotetext{
laborales o de proteger la seguridad y la salud laboral" OLARTE ENCABO, S., "La discriminación en materia de prevención de riesgos laborales, en AA. VV., El principio de igualdad en la negociación colectiva ..., o.c., p. 378.

${ }^{35}$ GARRIGUES JIMÉNEZ, A., "Las nuevas relaciones de trabajo: una perspectiva de género", en Revista de treball, economia i societat, 92 (2019).

${ }^{36}$ OIT. Estrategia global en materia de seguridad y salud en el trabajo. Conclusiones adoptadas por la Conferencia Internacional del Trabajo en su 91 ${ }^{\mathrm{a}}$ reunión, 2003, disponible en: http://www.ilo.org/wcmsp5/groups/public/---ed_protect/---protrav/---safework/documents/policy /wcms_154865.pdf.
} 
Sería necesario erradicar la idea de que las mujeres desarrollan sus trabajos en entornos más seguros o con ausencia de riesgos. No debe seguir utilizándose un enfoque neutral en la realización de las evaluaciones de riesgos laborales ya que los riesgos a los que están expuestas las trabajadoras quedan infravalorados ${ }^{37}$; tanto hombres como mujeres se enfrentan a diferentes riesgos en sus puestos de trabajo, o un mismo riesgo puede tener un efecto o consecuencia diferente por razón del género.

En la integración de la perspectiva de género en la prevención de riesgos laborales pueden destacarse como pautas o aspectos fundamentales para su consecución:

En primer lugar, la de investigar mejor los requisitos, condiciones y características de las ocupaciones, al objeto de identificar posibles riesgos para la salud, diferenciándolos y desagregándolos por razón de género. La práctica de la prevención debe contemplar todos los puestos de trabajo, todos los riesgos (seguridad, higiénicos, ergonómicos y psicosociales) y todos los daños (accidentes de trabajo, enfermedades profesionales y enfermedades relacionadas con el trabajo $^{38}$.

En segundo lugar, mejorar el registro de enfermedades profesionales. La salud de hombres y mujeres es diferente y desigual; es diferente, porque existen una serie de factores biológicos (genéticos, hereditarios, fisiológicos, etc.) que inciden en el riesgo de enfermar de forma distinta por razón de género, y es desigual porque existe otra serie de condicionantes sociales, explicados por el género, que influyen de forma injusta sobre la salud de hombres y mujeres ${ }^{39}$.

En tercer lugar, un desafío importante también sería el de reflexionar sobre la necesidad de adaptar el sistema de prevención de riesgos laborales de la empresa a la evolución de la tecnológicas y a la aparición de nuevas formas de trabajo flexibles desde una perspectiva de género. La realidad actual es que se avanza a pasos agigantados hacia un mercado de trabajo en el que cada vez más los requerimientos físicos, sensoriales o de atención van a ser asumidos por procesos automatizados, robotizados y digitalizados y donde el papel de la mujer está pendiente de configuración.

${ }^{37}$ UGT. Observatorio de riesgos psicosociales. La perspectiva de género en la prevención de riesgos laborales, Secretaría de Salud Laboral y Medio ambiente UGt-CEC, Madrid, 2019, pág. 9. Disponible en: http://www.observatorioriesgospsicosociales.com/sites/default/files/ publicaciones/La\%20Perspectiva\%20de\%20Genero.pdf.

${ }^{38}$ LINARES, P., "Repensar la prevención de riesgos laborales: algunas propuestas para el futuro", en Revista de Salud Laboral ISTAS- CCOO, 75 (2017).

${ }^{39}$ Ibidem. 
Por otra parte, una mayor incorporación de la mujer al mercado de trabajo puede generar que el porcentaje de mujeres cotizantes se iguale con el del hombre; incluso una mayor esperanza de vida de las mujeres, podría producir un incremento del coste, no sólo de las pensiones, sino también del resto de las prestaciones. La edad no debe considerarse como un problema sino una oportunidad; en la medida en que se combinen con políticas de mejora en las calificaciones laborales y de desarrollo productivo, se tendría un escenario de crecimiento que podría reforzarse mutuamente y garantizar una mejora en la prevención de riesgos profesionales. Así pues, resulta necesario realizar esfuerzos continuos para mejorar la cobertura de protección social y la prevención de los riesgos laborales, que hasta la fecha afecta de modo desigual a hombres y mujeres.

El insuficiente tratamiento normativo existente al respecto, dificulta que se articulen medidas de protección adecuadas frente a las situaciones de riesgo que pueden afectar a las mujeres de manera particular, bien sea por las propias circunstancias físicas en las que se encuentran, o bien por su presencia mayoritaria en determinados sectores u ocupaciones. La segregación ocupacional y la focalización del empleo femenino en el sector terciario son factores que no solo permiten afirmar la existencia de riesgos con mayor impacto en las mujeres frente a los hombres, sino que evidencian diferentes patrones de accidente y de enfermedad profesional.

La integración de la perspectiva de género en la prevención de riesgos laborales pasa necesariamente por investigar las áreas relevantes para las mujeres trabajadoras, que hasta ahora no han sido abordadas de forma suficiente, no subestimar los riesgos laborales a los que se exponen, reducir su falta de participación en las decisiones sobre salud laboral y garantizar que las medidas preventivas adoptadas sean apropiadas y eficaces ${ }^{40}$.

En definitiva, la mirada de género debe incorporarse en el análisis de las condiciones de trabajo, en la identificación de los riesgos, en su exposición y en los efectos en la salud y seguridad.

\subsection{La negociación colectiva como herramienta clave para su implantación}

La necesidad del enfoque de género en el análisis y en la regulación tanto legal como convencional- de la protección frente a los riesgos laborales ha quedado acreditada ${ }^{41}$; dicha orientación hacia la especificidad de género

\footnotetext{
${ }^{40}$ AA. VV., Estudio realizado por el Instituto Vasco de Seguridad y Salud Laborales (OSALAN). Pautas para la integración de la perspectiva de género, 2017, p. 8.

${ }^{41}$ Desde una perspectiva comparada, véase, NUNIN, R., "Género y prevención de riesgos laborales: las intervenciones de legislación y negociación colectiva en la experiencia italiana",
} 
puede llevarse a cabo tanto por medio de la negociación colectiva como por el legislador, pero en cualquier caso, se trata de una necesidad urgente ${ }^{42}$.

A pesar de estas bases -tanto legales como de políticas de acción-, aún no puede hablarse de mejoras normativas en el Derecho español que recojan la integración de la dimensión de género en los riesgos laborales en general, ni en los psicosociales, en particular, ni tampoco la adopción de estrategias preventivas específicas por razón de género ${ }^{43}$.Teniendo en cuenta que la repercusión del trabajo en la salud no es igualitaria entre ambos sexos por razones biológicas y sociales, las medidas de prevención de salud y protección social tampoco debieran ser idénticas ${ }^{44}$.

El legislador no puede limitarse a que las medidas de protección y prevención por razón de género se circunscriban a la maternidad de la mujer trabajadora, sino que ha de considerarse el género como factor de riesgo global, valorando los posibles peligros que para el trabajo genera la condición femenina. En este sentido, las evaluaciones de género constituyen una herramienta importante y el papel de la negociación colectiva en la identificación de las diferencias o desigualdades de género y su prevención también resultará esencial.

Para que las políticas de promoción de la salud y, por tanto, de minoración de los riesgos sean eficaces y garanticen la igualdad de género, habría que implementar una serie de medidas, entre las que cabría destacar:

- Una primera medida sería fortalecer el papel activo de la negociación colectiva que en la actualidad tiene un gran déficit en la adecuada implementación de la perspectiva de género en materia preventiva puesto que no está presente ni en la evaluación de riesgos a los que pueden quedar

en AA. VV., Género y no discriminación: Análisis transversal e interdisciplinar (Coord. Martha Elisa Monsalve Cuéllar), 2019, pp. 675-69.

${ }^{42}$ Sobre el alcance de protección de los riegos profesionales en la negociación colectiva, véanse, GIMENO DÍAZ DE ATAURI, P., "Contingencias profesionales y género en la negociación colectiva sectorial", en Revista Información Laboral, 2 (2018) 11. TOSCANI GIMÉNEZ, D., "La prevención de riesgos laborales en los convenios colectivos", en Gestión práctica de riesgos laborales: Integración y desarrollo de la gestión de la prevención, 46 (2008) 30-39.

${ }^{43}$ Sobre el particular, vid., in extenso, GARRIGUES GIMÉNEZ, A., "Hacia un nuevo paradigma (no androcéntrico) en la prevención de riesgos laborales: la necesaria e inaplazable integración normativa y técnica del diferencial de sexo y de género", en Derecho de las Relaciones Laborales, 8 (2017) 763-785, y también véase, "Las nuevas relaciones de trabajo: una perspectiva de género..., o.c., p. 23.

${ }^{44}$ RUIZ-RICO RUIZ, C., "La desprotección del derecho constitucional a la salud desde una perspectiva de género como causa de responsabilidad sanitaria", en La Ley (2011), (LA LEY 14173/2011). 
expuestas las trabajadoras, ni tampoco lo está en el plan de prevención que habrá de implementarse en la empresa, aspecto que habría que cambiar ${ }^{45}$.

- En la búsqueda de soluciones reales y efectivas va a resultar esencial la participación e implicación de las mujeres en el proceso de evaluación y reflejo de sus opiniones, experiencias, conocimientos y capacidades. Y es que la escasa participación de las mujeres en todos los niveles de consulta y de toma de decisiones en materia de seguridad y salud está contribuyendo a que se preste menos atención a sus necesidades y a que la evaluación de los riesgos sea deficiente.

- Resulta necesario elaborar políticas eficaces de seguridad y salud en el trabajo, tanto nacionales como internacionales, basadas en información más precisa acerca de la relación entre la salud y el género. El papel de la negociación colectiva en la identificación de las diferencias o desigualdades de género y su prevención también resultará esencial, aunque ello sin olvidar también, como algunos autores han señalado, el papel fundamental del legislador, toda vez que nos encontramos ante una materia en la que el Estado está llamado a desempeñar un papel protagonista a través de normas de Derecho necesario absoluto, frente al papel secundario del convenio colectivo como fuente de regulación ${ }^{46}$.

En lo que refiere a la negociación colectiva, a pesar de que casi todos los convenios colectivos contienen cláusulas relativas a la prevención de riesgos laborales o a la seguridad y salud de los trabajadores, predominan aquéllas cláusulas que se limitan a la remisión a lo dispuesto en la LPRL o a la reproducción de los preceptos legales. En lo que refiere al género, las cláusulas existentes son generalistas o inespecíficas, y se limitan a referirse a la protección por razón de embarazo y maternidad ${ }^{47}$, por ello algunos autores aluden al carácter indiferenciado de los riesgos en función del género ${ }^{48}$. Otras cláusulas, por el contrario, permiten definir y delimitar su contenido y ámbito de aplicación personal. A este respecto, destaca el plan de igualdad del Convenio colectivo grupo Eroski ${ }^{49}$ que contempla

${ }^{45}$ Véase, en este sentido, NIETO ROJAS, P., "Género, Prevención de Riesgos y Negociación colectiva", $2017 \ldots$, o.c., p. 3.

${ }^{46}$ NIETO ROJAS, P., "Dimensión de género en el tratamiento convencional de la seguridad y salud laboral", en AA. VV., Informe sobre salud laboral desde la perspectiva de género (Dir. E. M. ${ }^{\text {a }}$ Blázquez Agudo). Instituto de estudios de género. UC3M, Edición digital, Madrid 2017, p. 76.

${ }^{47}$ En este sentido, vid. FERNÁNDEZ DOCAMPO, B., "La salud laboral desde una perspectiva de género", en VV. AA. (coord. Lousada Arochena), El principio de igualdad en la negociación colectiva, Comisión Consultiva Nacional de Convenios Colectivos, Madrid 2008, p. 330.

${ }^{48}$ OLARTE ENCABO, S., “La discriminación por razón de género"..., o.c., p. 382.

${ }^{49}$ Resolución de 14 de febrero de 2018, de la Dirección General de Empleo, por la que se registra y publica el VI Convenio colectivo de supermercados del Grupo Eroski. BOE 2 de febrero de 2018. 
de forma diferenciada los riesgos músculo esqueléticos, que generan patologías sobre todo en las trabajadoras, incluyendo en las revisiones médicas pruebas de musculatura y esqueleto -Protocolos de Reconocimientos Médicos Específicos Musculo-Esqueléticos-. Como se ha señalado, este tipo de previsión, al tener en cuenta la dimensión de género, es un avance en la consideración de riesgos que afectan a mujeres y la lucha contra la discriminación en materia preventiva, siendo especialmente positivo el que se tengan en cuenta los riesgos músculo esqueléticos en trabajos distintos a los de carga de peso -predominantemente masculinos-, tales como los que comportan una postura forzada -cajeros/asque son desempeñados mayoritariamente por trabajadoras ${ }^{50}$.

El análisis de la negociación colectiva evidencia, sin embargo, que son aún pocos los convenios que prevén en su articulado políticas de género en materia de prevención y los escasos convenios que sí lo hacen apenas aportan especial relevancia, toda vez que se limitan a recoger en su articulado meras declaraciones programáticas. A juicio de la doctrina esta carencia se manifiesta en la escasa consideración en cualquiera de las actividades preventivas de la incidencia de la «doble jornada» sobre las mujeres en el trabajo y de la mayor precariedad laboral sobre las mujeres, pero también por el desconocimiento de riesgos genéricos con especial incidencia en mujeres trabajadoras y de riesgos específicos ${ }^{51}$. A pesar de que no se están cumpliendo las expectativas previstas, se espera que, a medio plazo, la negociación colectiva sea mucho más prolija.

No obstante, como ejemplo de buenas prácticas cabría mencionar y solo en un sector tan feminizado como es el de la enseñanza privada la creación de un servicio especializado en enfermedades profesionales, en concreto, para las enfermedades propias de este sector -entre ellas, las neurológicas crónicas, patologías otorrinolaringológicas, enfermedades infecto-contagiosas crónicas o alergias crónicas ${ }^{52}$-, al que se le encomienda un análisis de estos riesgos desagregado por sexo. A pesar de estas buenas prácticas, son muy pocos los convenios que atienden a la existencia de riesgos exclusiva o mayoritariamente femeninos ${ }^{53}$.

En definitiva, las fórmulas convencionales serán, como puede imaginarse, muy diversas, como también lo son su presentación, ubicación y tratamiento. De

${ }^{50}$ OLARTE ENCABO, S., “La discriminación por razón de género”..., o.c., p. 386.

${ }^{51}$ OLARTE ENCABO, S., o.c., p. 383.

52 Resolución de 2 de julio de 2018, de la Dirección General de Trabajo, por la que se registra y publica el Convenio colectivo nacional de centros de enseñanza privada de régimen general o enseñanza reglada sin ningún nivel concertado o subvencionado. BOE 11 de julio de 2018.

${ }^{53}$ NIETO ROJAS, P., "Dimensión de género en el tratamiento convencional de la seguridad y salud laboral"..., o.c., p. 81. En el mismo sentido FERNÁNDEZ DOCAMPO, B., "La salud laboral desde una perspectiva de género"..., o.c., p. 343. 
momento, lo que abundan son las declaraciones programáticas, los enunciados de principios generales y las meras declaraciones de intenciones sin esbozar siquiera -salvo puntuales excepciones- mandatos más allá de los ya previstos legalmente.

\section{CONSIDERACIONES FINALES}

La prevención de riesgos laborales en la empresa no puede ser aplicada de la misma forma a todos los trabajadores, sino que tiene que ser adaptada a las características biológicas y sociales diferenciales por razón de género.

El futuro de la prevención de riesgos laborales y, en particular, por razón de género continúa siendo una asignatura pendiente. Quizá sea el momento de plantearse que la proyección normativa de futuro pase por la intervención del legislador de forma que la perspectiva de género y su protección y prevención tenga reflejo específico en la Ley de Prevención de Riesgos Laborales, así se aportaría mayor seguridad para las partes de la relación laboral en sus obligaciones y derechos de forma que permitan garantizar un régimen jurídico seguro y suficiente.

La necesidad de un enfoque de género en el análisis y en la regulación -tanto legal como convencional- de la protección frente a los riesgos laborales más allá de la maternidad ha quedado acreditada; dicha orientación hacia la especificidad de género puede llevarse a cabo tanto por medio de la negociación colectiva como por el legislador; en cualquier caso, de lo que no hay duda es que se trata de una necesidad urgente.

\section{BIBLIOGRAFÍA}

- AA. VV., Informe sobre salud laboral desde la perspectiva de género, (Dir. E. Blázquez Agudo), Instituto Estudios de Género. Universidad Carlos III de Madrid, Getafe 2017.

- AA. VV., Estudio realizado por el Instituto Vasco de Seguridad y Salud Laborales (OSALAN). Pautas para la integración de la perspectiva de género, 2017.

- ACALE SÁNCHEZ, M., "Género y siniestralidad laboral: causa y efecto", en Gestión Práctica de Riesgos Laborales, 69 (2010) 27.

- BLÁZQUEZ AGUDO, E., "La necesaria asunción de la prevención de riesgos laborales desde una perspectiva de género", en Femeris, 5 (2020) 5. 
- COPSEY, S., "Trabajo más seguro y saludable a cualquier edad", en AA:VV El Envejecimiento de la Población Trabajadora Balance crítico de la situación y propuestas de mejora. Observatorio Vasco sobre Acoso y Discriminación, Bizkaia 2018.

- FERNÁNDEZ DOCAMPO, B., "La salud laboral desde una perspectiva de género", en VV. AA. (coord. Lousada Arochena), El principio de igualdad en la negociación colectiva, Comisión Consultiva Nacional de Convenios Colectivos, Madrid 2008.

- GARCÍA GÓMEZ, M; CASTAÑEDA LÓPEZ, R.; HERRADOR ORTIZ, Z.; LÓPEZ MENDUIÑA, P.; MONTOYA MARTÍNEZ, L.M; ÁLVAREZ MAEZTU, E., et Al., Estudio epidemiológico de las enfermedades profesionales en España (1990 - 2014). Madrid, Ministerio de Sanidad, Servicios Sociales e Igualdad, 2017.

- GARCÍA GÓMEZ, M., y CASTAÑEDA LÓPEZ, R., "Enfermedades profesionales declaradas en hombres y mujeres en España en 2004", en Revista Española de. Salud Publica, 4 (2006).

- GARRIGUES GIMÉNEZ, A., "Hacia un nuevo paradigma (no androcéntrico) en la prevención de riesgos laborales: la necesaria e inaplazable integración normativa y técnica del diferencial de sexo y de género", en Derecho de las Relaciones Laborales, 8 (2017).

- GARRIGUES GIMÉNEZ, A., "Las nuevas relaciones de trabajo: una perspectiva de género", en Revista de treball, economia $i$ societat, 92 (2019).

- GIL PÉREZ, M. E., "Mujer y salud laboral”, en AA. VV., Mujer y trabajo, Bomarzo, Albacete 2003.

- GIMENO DÍAZ DE ATAURI, P., "Contingencias profesionales y género en la negociación colectiva sectorial”, en Revista Información Laboral, 2 (2018).

- GONZÁLEZ GÓMEZ, Ma F., "Salud laboral y género. Apuntes para la incorporación de la perspectiva de género en el ámbito de la prevención de riesgos laborales", en Revista Medicina y Seguridad en el Trabajo, 57 (2011).

- GONZÁlEZ ORTEGA, S., y BARCELÓN COBEDO, S., “Cien años de promoción de la Seguridad Social. El papel de la OIT en la creación, expansión, perfeccionamiento y adaptación de los sistemas de protección social", en Revista Trabajo y Derecho, 9 (2019). 
- GONZÁLEZ SEGARRA, F.J., Riesgos ergonómicos y psicosociales en los sectores feminizados de Andalucía. Ed. UGT-Andalucía, Sevilla 2006.

- LINARES, P. "Repensar la prevención de riesgos laborales: algunas propuestas para el futuro", en Revista de Salud Laboral ISTAS- CCOO, 75 (2017).

- MARTÍNEZ YÁÑEZ, N. M., "Algunas consideraciones sobre igualdad por razón de género en el marco de la seguridad y salud en el trabajo", en Lan Harremanak, 25 (2012).

- NIETO ROJAS, P., "Dimensión de género en el tratamiento convencional de la seguridad y salud laboral" en AA.VV., Informe sobre salud laboral desde la perspectiva de género (Dir. E. M.', Blázquez Agudo). Instituto de Estudios de Género. UC3M. Edición digital, Madrid 2017.

- NIETO ROJAS, P., "Género, Prevención de Riesgos y Negociación colectiva”, en Revista de información laboral, 9 (2017). (BIB 2017\13141).

- NUNIN, R., "Género y prevención de riesgos laborales: las intervenciones de legislación y negociación colectiva en la experiencia italiana", en AAVV. Género y no discriminación: Análisis transversal e interdisciplinar (Coord. Martha Elisa Monsalve Cuéllar), 2019.

- OLARTE ENCABO, S., "La discriminación en materia de prevención de riesgos laborales, en AA.VV., El principio de igualdad en la negociación colectiva, (Dir. C. Sánchez Trigueros), Ministerio de Empleo y Seguridad Social, Madrid 2016.

- PÉREZ DEL RÍO, T., y BALLESTER PASTOR, M. A., Mujer y salud laboral, La Ley, Las Rozas, Madrid 1999.

- RIVAS VALLEJO, P., "La prevención de riesgos desde una perspectiva de género: posibilidades y realidades", en Anuario de relaciones laborales en España: objetivo el trabajo, coord. por Antonio Ferrer Sais; Santos M. Ruesga Benito (ed. lit.), Carlos Resa Nestares (ed. lit.), 2 (2011).

- RIVAS VALLEJO, P., "Salud y género: perspectiva de género en la salud laboral", en Revista del Ministerio de Trabajo y Asuntos Sociales, 74 (2008).

- RUIZ-RICO RUIZ, C., "La desprotección del derecho constitucional a la salud desde una perspectiva de género como causa de responsabilidad sanitaria", en La Ley (2011), (LA LEY 14173/2011). 
- TOSCANI GIMÉNEZ, D., "La prevención de riesgos laborales en los convenios colectivos", en Gestión práctica de riesgos laborales: Integración y desarrollo de la gestión de la prevención, 46 (2008).

- UGT, Guía de Calificación jurídica de las patologías causadas por Riesgos Psicosociales en el trabajo. Propuesta de mejora 2018. 
